\title{
FORMACIÓN NOGONTOVA, UNA NUEVA UNIDAD LITOESTRATIGRÁFICA EN LA CORDILLERA ORIENTAL DE COLOMBIA
}

\author{
Mario Moreno-Sánchez ${ }^{1}$; Luz Mary Toro-Toro ${ }^{1}$; Arley Gómez-Cruz ${ }^{1}$; Elvira Cristina Ruiz J. ${ }^{1}$
}

DOI: http://dx.doi.org/10.18273/revbol.v38n2-2016003 (c) (1) $\Theta$

Forma de citar: Moreno-Sánchez, M., Toro-Toro, L.M., Gómez-Cruz, A., y Ruiz, E.C. 2016. Formación Nogontova, una nueva unidad litoestratigráfica en la Cordillera Oriental de Colombia. Boletín de Geología, 38(2): 55-62.

\section{RESUMEN}

La nueva unidad de lavas almohadillados que afloran en la vereda Nogontova (Boyacá) y San José de Miranda (Santander), flanco occidental de la Cordillera Oriental, está constituida por basaltos con texturas porfiríticas y amigdalares, con microfenocristales de plagioclasa y clinopiroxeno embebidos en una masa fundamental vitrofídica, microlítica e intergranular. La edad de esta unidad se estima en un rango de Triásico Tardío al Jurásico.

Palabras clave: basaltos almohadillados, petrografía, Cordillera Oriental.

\section{NOGONTOVA FORMATION, A NEW LITHOSTRATIGRAPHIC UNIT IN THE EASTERN CORDILLERA OF COLOMBIA}

\begin{abstract}
The new unit of pillow lavas outcropping in the Nogontova area (Covarachía, Boyacá) and San Jose de Miranda (Santander), western flank of the Cordillera Oriental, is formed of basalts with porfidic and vesicular texture, with microcrystals of plagioclase and clinopyroxen embedded in an intergranular microlithic vitrophyric groundmass. The age of this unit is estimated to range from Late Triassic to Jurassic.
\end{abstract}

Keywords: pillow basalts, petrography, Eastern Cordillera

\footnotetext{
${ }^{1}$ Departamento de Ciencias Geológicas Universidad de Caldas. Manizales, Colombia; mario.moreno@ucaldas.edu.co, luz. toro@ucaldas.edu.co; arley.gomez@ucaldas.edu.co,elvira.ruiz@ucaldas.edu.co
} 


\section{INTRODUCCIÓN}

Colombia puede ser dividida en dos grandes dominios geológicos: el occidental, al oeste de la Cordillera Central, conformado por un basamento oceánico cubierto por secuencias sedimentarias cretácicas, asociadas frecuentemente con basaltos almohadillados; por otro lado, el dominio oriental, al este de la Cordillera Central, constituido por un basamento de origen continental donde las rocas ígneas básicas mesozoicas no son comunes.

En la Cordillera Oriental y la Sierra de Perijá se presentan exposiciones de rocas volcánicas Jurásicas; Maze (1984) describe flujos de basalto-andesita en la formación La Quinta en la Serranía de Perijá, Ward et al. (1973) reconoce riolitas en la Formación Bocas y diques básicos en la Formación Jordán; menciona además la presencia de diques básicos (basaltos y diabasas) afectando rocas metamórficas e ígneas entre Rionegro y Bucaramanga. En el Valle Superior del Magdalena, Serranía de San Lucas y Sierra Nevada de Santa Marta se reportan rocas volcánicas básicas e intermedias de edad Jurásica que se han interpretado como el producto de extensión cortical relacionada a la apertura de un rift activo desde el Triásico Tardío hasta el Jurásico (Kammer and Sánchez, 2006; Mojica y Franco, 1992).

En este trabajo se establece la nueva unidad litoestratigráfica denominada Formación Nogontova, expuesta al sur del Macizo de Santander, en ambas márgenes del río Chicamocha, entre los departamentos de Boyacá y Santander. Esta formación está constituida por lavas basálticas que frecuentemente muestran estructuras peperíticas, almohadilladas y amigdalares, indicando enfriamiento subacuático de baja profundidad (Jones, 1969). De esta manera, la Formación Nogontova se constituyen en la primera unidad de rocas básicas efusivas con este tipo de estructura reportada en la Cordillera Oriental colombiana. La morfología almohadillada y amigdalar de los basaltos es la principal característica que permite su clasificación primaria en campo. Los basaltos de la Formación Nogontova, por sus estructuras almohadilladas y peperíticas, no tienen antecedentes históricos en la Cordillera Oriental.

\section{MARCO GEOLÓGICO REGIONAL}

El área del presente estudio se ubica al sur del Macizo de Santander en la Cordillera Oriental. El basamento metamórfico está constituido por el Neis de Bucaramanga (Goldsmith et al., 1971) y la Formación Silgará (Ward et al., 1973), con edades que varían entre el Neoproterozoico y el Ordovícico. El basamento está cubierto por secuencias levemente metamorfoseadas del Paleozoico tardío denominadas Formación Floresta, "Floresta Metamorfoseada" (Ward et al., 1973) y Metasedimentitas de Guaca (Royero y Vargas, 1999). El área está intruida por un grupo de cuerpos plutónicos e hipoabisales de carácter intermedio a básico cuyas edades van desde el Precámbrico (EtayoSerna et al., 1986) hasta el Cretácico (Ward et al., 1973).

El lapso Triásico - Jurásico está registrado en el Macizo de Santander por rocas sedimentarias de origen continental y cuerpos intrusivos de carácter intermedio. La Formación Bocas (Dickey, 1941; Ward et al., 1973) constituida por sedimentitas y vulcanitas continentales del Jurásico Temprano (Remy et al., 1975) se deposita sobre un basamento constituido por metamorfitas del Neis de Bucaramanga y los esquistos de la Formación Silgará. La Formación Jordán (Cediel, 1968) del Jurásico Temprano está constituida por tobas, limolitas rojas y areniscas gris verdosas de origen continental. Hacia la base de la unidad se reportan conglomerados con clastos del granito de Pescadero de edad Jurásico Temprano (Goldsmith et al., 1971; Ward et al., 1973). La Formación Girón (Langenheim, 1954; Ward et al., 1973), discordante sobre la Formación Jordán, es una unidad de espesores variables que en el área está constituida por areniscas y conglomerados arcósicos con niveles de limolitas rojizas de origen continental. La datación de circones detríticos (Horton et al., 2010) sugiere que la depositación de la Formación Girón es posterior al Jurásico Temprano. La secuencia del cretácico basal se inicia con las cuarzoarenitas de las formaciones Río Negro (Hedberg, 1931) y Los Santos (Formación Tambor sensu Morales et al., 1958); la sucesión superior está constituida por calizas y lodolitas de origen marino.

\section{METODOLOGÍA}

Los contactos y datos de campo se georreferenciaron con la ayuda de un Garmin GPSMAP 64s. Se recolectaron muestras de roca representativa de la unidad y se analizaron las secciones delgadas correspondientes; éstas se clasificaron de acuerdo con Le Maitre et al. (2002).

\section{FORMACIÓN NOGONTOVA}

El nombre de la unidad proviene de la vereda Nogontova al norte del municipio de Covarachía en el departamento de Boyacá, en donde afloran los basaltos. La unidad aflora en ambas márgenes del río Chicamocha, que en el área es el límite natural entre los departamentos 
de Santander y Boyacá. La formación se ubica en la plancha IGAC 136-III-B. La sección tipo se sitúa en el camino que de San José de Miranda conduce al sitio de La Burrera, en el margen norte del río Chicamocha (FIGURA 1).

Los basaltos en superficie abarcan sectores de los municipios de Molagavita y San José de Miranda (vereda Cucharito) en el departamento de Santander y del municipio de Covarachía en el departamento de Boyacá.
La Formación Nogontova se dispone discordantemente sobre las metasedimentitas paleozoicas ("Paleozoico indiferenciado" en este trabajo), unidad cuyo nombre varía según diferentes autores: "Formación Floresta" por Acosta y Solano (1999), "Metasedimentitas de Guaca" por Royero y Vargas (1999) y "Floresta Metamorfoseado" por (Ward et al., 1973). La unidad es suprayacida discordantemente por sedimentitas mesozoicas que incluyen conglomerados rojos, conglomerados de cuarzo y cuarzoarenitas de la Formación Río Negro (sensu Vargas et al., 1984).

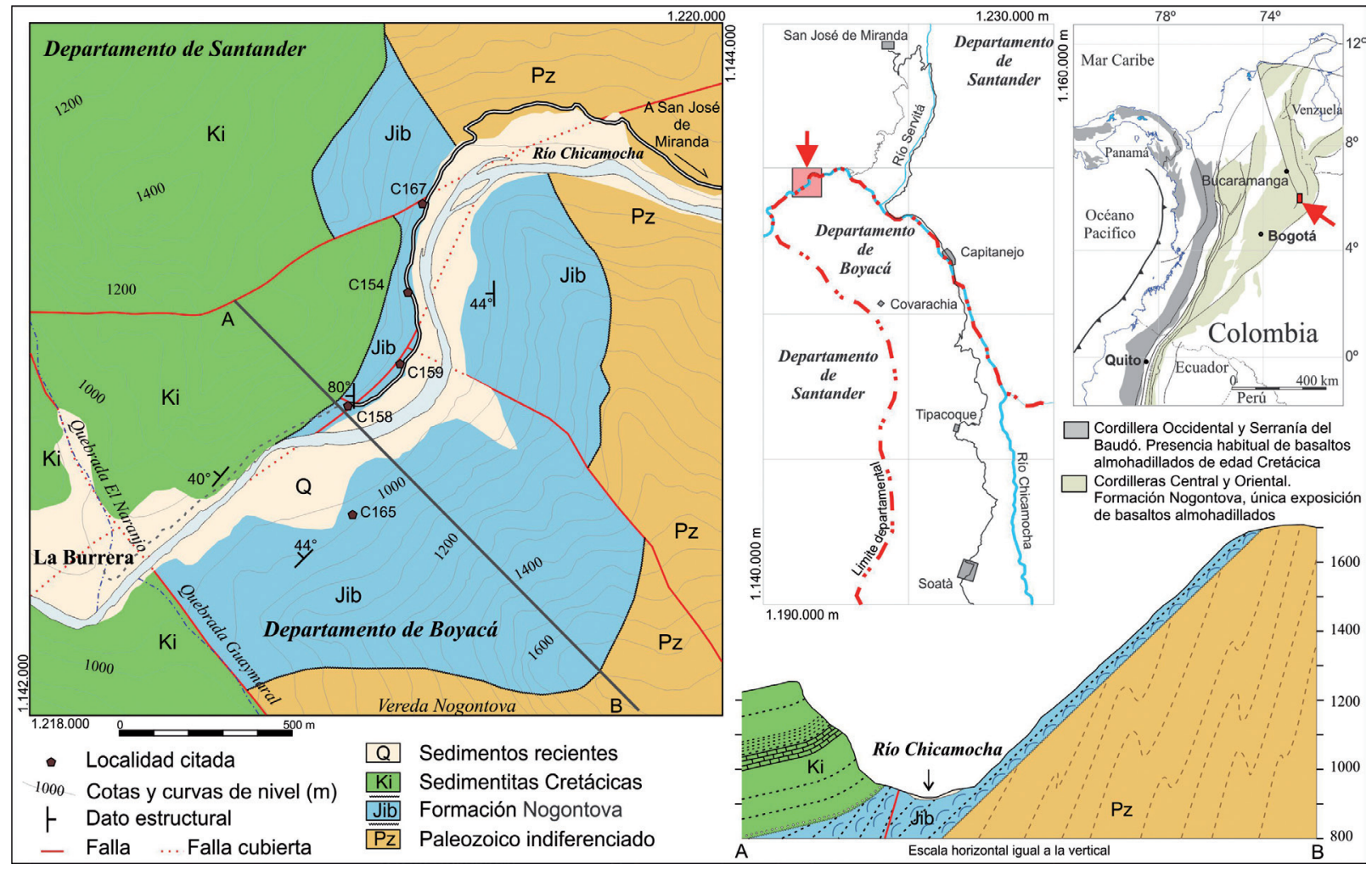

FIGURA 1. Mapa geológico de la sección tipo y ubicación geográfica de los basaltos de la Formación Nogontova entre los departamentos de Boyacá y Santander.

La unidad en la sección tipo está constituida de base a techo por una serie de coladas lávicas de potencia variable que generan superficies escarpadas fácilmente discernibles en el terreno. En los sectores donde las coladas basálticas entran en contacto con los sedimentos se desarrollan peperitas que se presentan como brechas cementadas por carbonatos y como inyecciones de sedimentos entre las almohadillas (FIGURAS 2 y 3 ). En la parte media y basal de la unidad existen algunos niveles discontinuos subtabulares, de no más de $4 \mathrm{~m}$ de espesor, de lodolitas negras laminadas.

El espesor medido de la unidad alcanza los 230 me en el sector central y se acuña erosivamente hacia el norte donde desaparece. Al suroeste la formación es cortada por la falla de la quebrada Guaymaral. Se propone que se denomine Formación Nogontova a las rocas volcánicas básicas que presenten las mismas características geológicas y disposición estratigráfica de la unidad definida en la sección tipo.

No se encontraron fósiles. Las coladas basálticas de la unidad se formaron en el intervalo entre la sedimentación de la Formación Río Negro ("Tambor" según Morales et al., 1958; Ward et al., 1973) y el metamorfismo de las metasedimentitas basales. La Formación Río Negro abarca el Berriasiano (o posiblemente Titoniano) hasta el Hauteriviano (Campbell, 1962) y el protolito de 
las metasedimentitas paleozoicas en el área podrían alcanzar el Carbonífero tardío con metamorfismo entre el Pérmico tardío y el Triásico basal (Moreno-Sánchez et al., 2005; Moreno-Sánchez et al., 2008). La Formación Girón (Jurásico Tardío), constituida por capas de arenisca y conglomerados rojos de origen continental, aflora en los alrededores de Molagavita, pero se adelgaza rápidamente al sur hasta casi desaparecer a 2,5 km al noreste de la localidad C167, en donde se observan algunos remanentes no cartografiables entre la Formación Rio Negro y los basaltos de la Formación Nogontova. La Formación Girón presenta guijos de rocas ígneas derivadas de cuerpos datados del Jurásico Temprano (Ward et al., 1973); por otro lado, zircones detríticos recolectados en esta formación indican edades máximas de depósito con una media de 184,5 $\pm 4,2 \mathrm{Ma}$ (Horton et al., 2010). Se infiere que el rango potencial de la Formación Nogontova está en el intervalo Triásico Tardío - Jurásico, siendo anterior a la Formación Girón y posterior a las unidades paleozoicas del área.

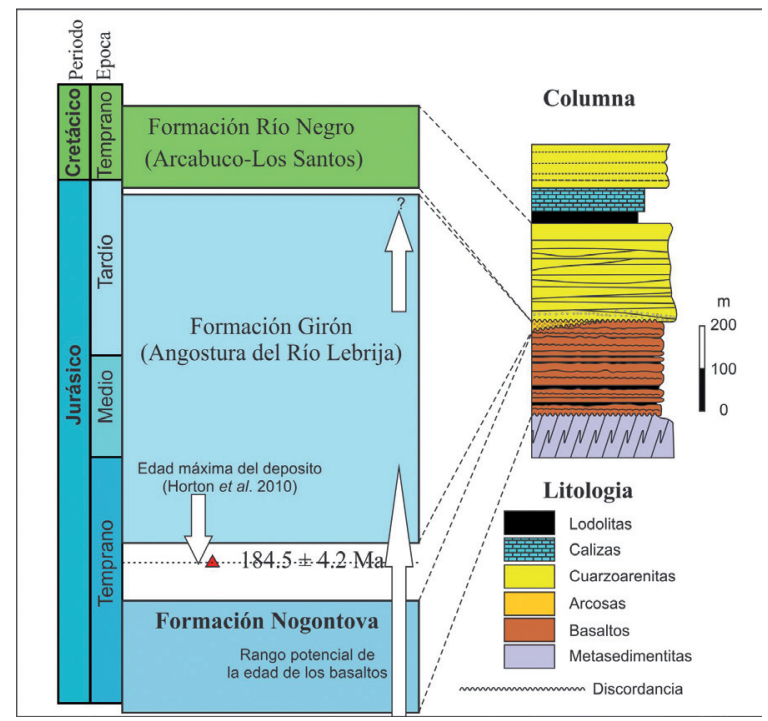

FIGURA 2. Esquema cronoestratigráfico y columna generalizada mostrando las relaciones estratigráficas con la Formación Nogontova.

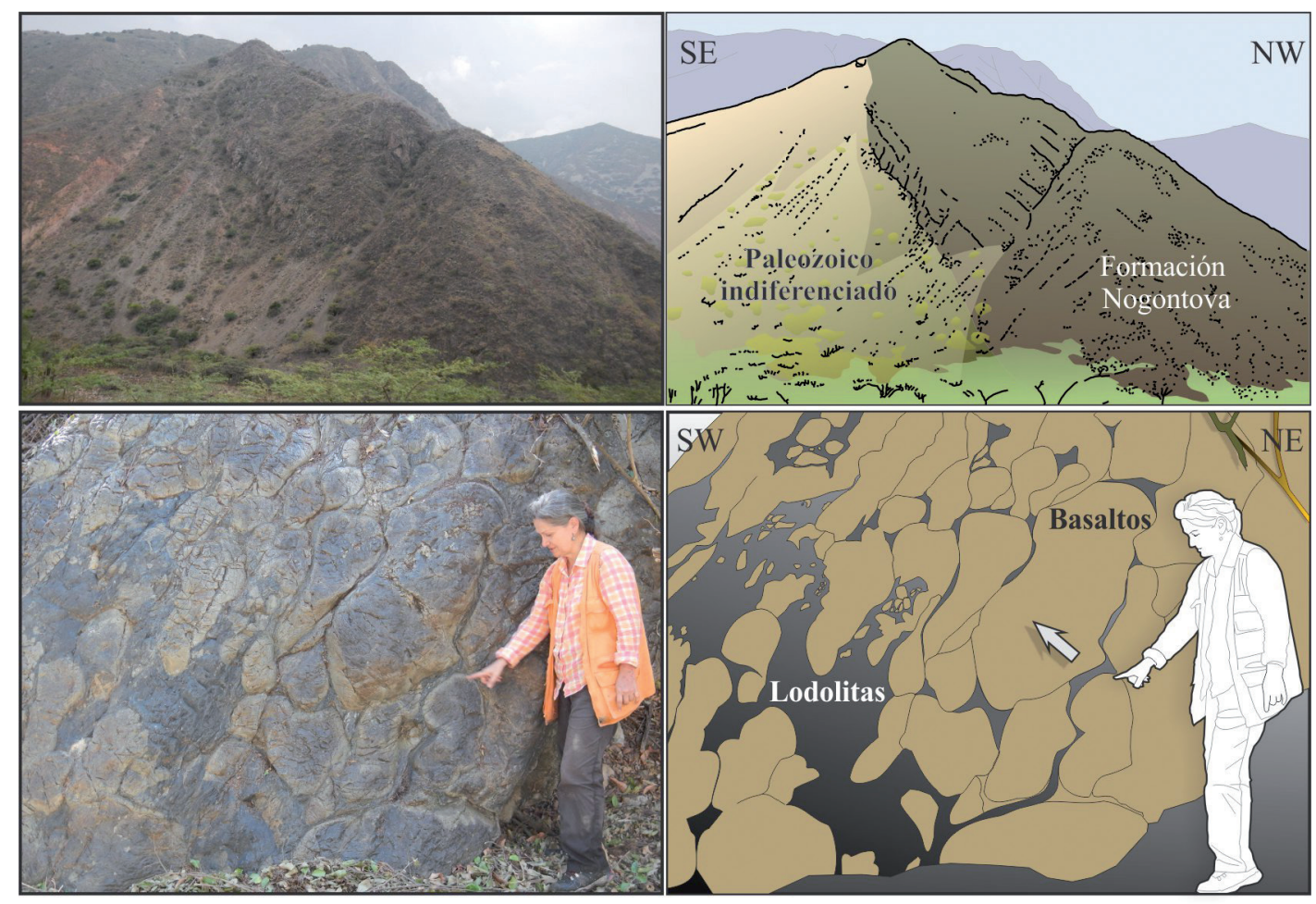

FIGURA 3. Arriba, aspecto de los basaltos de Nogontova en la ribera sur (departamento de Boyacá) del río Chicamocha. Abajo, morfología almohadillada de los basaltos de la Formación Nogontova sobre el camino que conduce a la hacienda La Burrera (departamento de Santander), sirve de referencia la profesora L.M. Toro-Toro.

\section{PETROGRAFÍA}

Las rocas estudiadas en este trabajo corresponden a basaltos con almohadillas con formas elípticas a subredondeadas, con diámetros medios entre 0,40 a 0,70 $\mathrm{m}$ con un empaquetamiento apretado. Hacia los bordes se observa una corteza vítrea discreta de pocos milímetros de espesor, aproximadamente de 5 a $8 \mathrm{~mm}$, coloración verde claro, indicando un enfriamiento rápido; ambas estructuras son fácilmente discernibles en los afloramientos. Las mayores dimensiones observadas van desde 0,30 a $1,5 \mathrm{~m}$ en el sentido de su eje mayor. El color de estas rocas es 
gris verdoso a verde oscuro con presencia de superficies de alteración castaño rojizas (FIGURA 3).

Los basaltos almohadillados presentan texturas porfiríticas definidas por microfenocristales de plagioclasa y cristales granulares y/o prismáticos de piroxenos contenidos en una masa fundamental intersectal, microlítica y amigdalar (FIGURAS 4A, 4B Y 4C). La masa fundamental está constituida por microlitos de plagioclasa, piroxeno granular que ocupa los intersticios entre los microlitos, opacos y vidrio. Los clinopiroxenos, con tamaños menores de $0,06 \mathrm{~mm}$, muestran relieve y birrefringencia amarilla del primer orden indicando una composición pigeonítica. La masa fundamental está constituida por vidrio de color café oscuro, desvitrificado y/o reemplazado por carbonatos y clorita/esmectita.
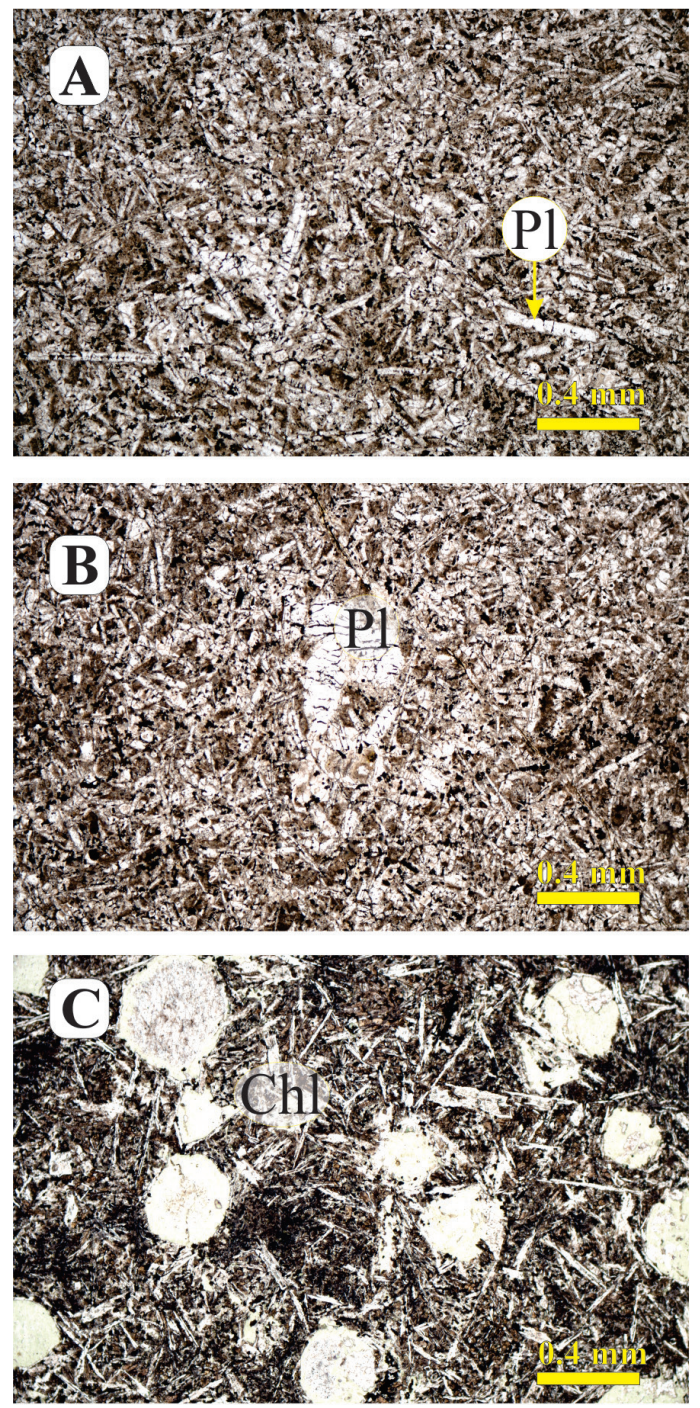

La plagioclasa es la fase mineral más abundante y ocurre como microlitos en cristales subhedrales con hábito tabular, tamaños menores de $0,1 \mathrm{~mm}$ y una disposición entrecruzada en donde los intersticios son ocupados principalmente por vidrio y/o piroxenos, presentan macla de carlsbad principalmente y albitacarlsbad. En general la plagioclasa es sustituida parcialmente por clorita y epidota, con morfologías de bordes corroídos y algunos microlitos desarrollan proyecciones fibrosas a partir de núcleos elongados en forma de tableta, esqueletal y acicular.

Los basaltos también presentan texturas amigdalares con tamaños variables desde $1 \mathrm{~mm}$ hasta $0,05 \mathrm{~mm}$, con formas redondeadas y ligeramente ovaladas, rellenas principalmente por carbonatos, clorita y sílice.
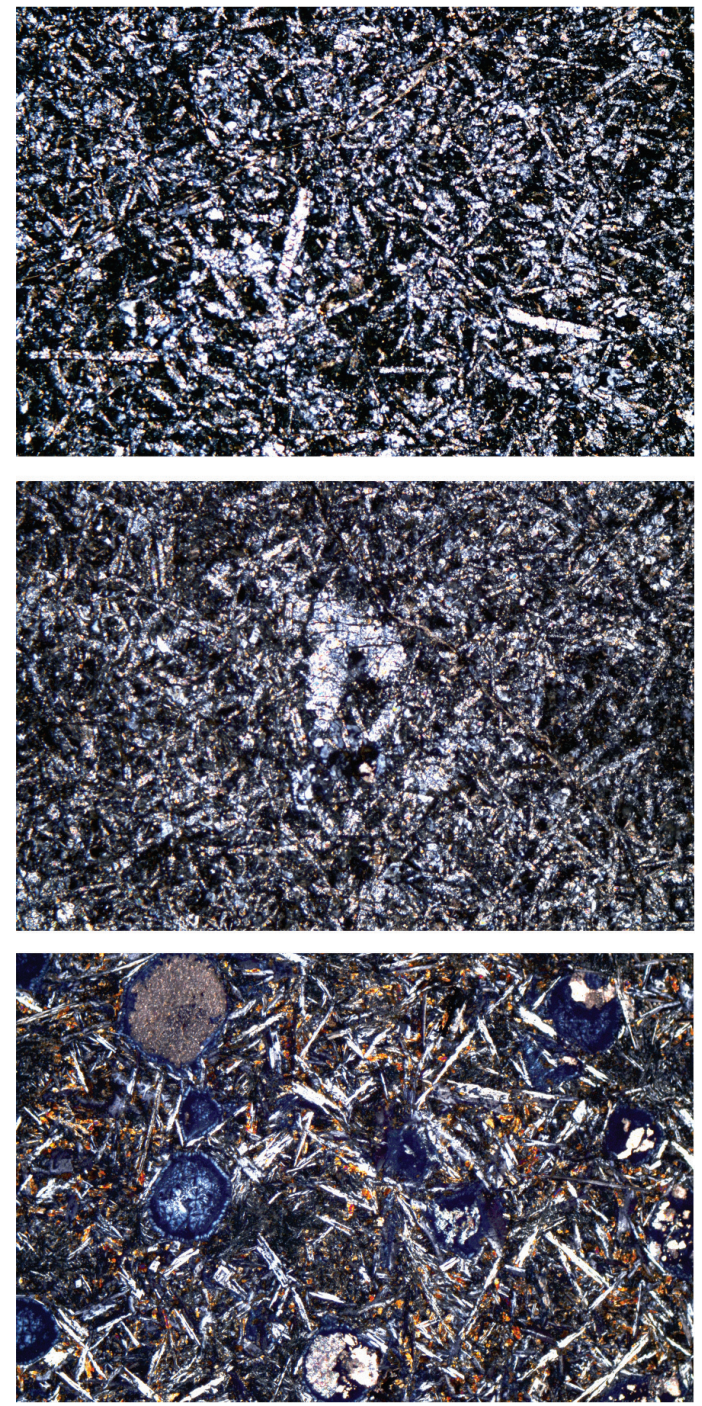

FIGURA 4. A. Basaltos con masa fundamental constituida por microlitos de plagioclasa (P1). B. Textura porfirítica definida por microfenocristales de plagioclasa $(\mathrm{Pl})$. C. Textura amigdalar, con formas redondeadas y ligeramente ovaladas, rellenas principalmente por carbonatos, clorita $(\mathrm{Chl})$ y sílice. 


\section{INTERPRETACION AMBIENTAL}

La Formación Nogontova se presenta como una serie de coladas basálticas subtabulares con intercalaciones discontinuas de lodolitas. Los basaltos se han alterado por la intervención de procesos hidrotermales de metamorfismo de fondo oceánico con posible efecto leve de un evento subsecuente de metamorfismo orogénico. Las coladas lávicas fluyeron bajo el agua en condiciones someras tal como lo atestigua la estructura almohadillada y la presencia de abundantes amígdalas en los basaltos (ver Jones, 1969; Moore, 1965; Moore and Schilling, 1973). Según Kennish and Lutz (1998), las coladas de estructura masiva (sheet flows) son el producto de lavas con mayor fluidez que las lavas con estructuras almohadilladas. Ballard et al. (1979) indica que, según lo observado en dorsales, las lavas almohadilladas son estados tardíos posteriores a los flujos masivos (sheet flows). El desarrollo de peperitas indica que los sedimentos blandos (con agua) fueron comprimidos y forzados a extruirse por el sobrepeso generado por el avance de las coladas basálticas. Entre los cojines se presentan ocasionalmente brechas con matriz calcárea que indican que una corteza de basalto sólido, enfriado bajo el agua, fue fragmentado durante el avance de flujos lávicos activos. La presencia de niveles lodolíticos intercalados con los basaltos y de sedimentos en los intersticios de los lóbulos de basaltos sugiere que las coladas fueron contemporáneas con la sedimentación.

\section{DISCUSIÓN-CONCLUSIONES}

A la fecha de este trabajo, la Formación Nogontova no tiene equivalente litológico en la Cordillera Oriental. Un elemento clave en la comparación de estos cuerpos básicos con otras unidades en el Macizo de Santander, es su edad y posición estratigráfica. La "Formación Floresta Metamorfoseada", sobre las cuales yacen los basaltos, incluye rocas de edad Carbonífero tardío, sugiriendo que el volcanismo que produjo los basaltos postdata esta edad.

La Formación Diamante del Pérmico (Dickey, 1941; Ward et al., 1973), aflorante en cercanías de Bucaramanga, exhibe secuencias calcáreas pérmicas sin volcanismo aparente. Al techo, sobre los basaltos se depositan discordantemente capas rojas (Formación Girón?) y cuarzo arenitas de la Formación Rio Negro del Cretácico basal. La edad de la Formación Girón se sitúa en el Jurásico Medio según datos de circones detríticos que aportan edades máximas de depósito con una media de 184,5 \pm 4,2 Ma (Horton et al., 2010). De esta manera el rango cronológico en donde se desarrolla la Formación Nogontova se sitúa entre el Triásico y el Jurásico Medio.
Así, se deben excluir de la comparación unidades ígneas paleozoicas del Macizo de Santander. En cercanías a Covarachía existen pequeños enclaves gabroicos, afectando ortoneises, que podrían ser coetáneos con los basaltos. Sin embargo, entre las unidades volcanosedimentarias en la Cordillera Oriental que podrían correlacionarse con los basaltos, solo pueden ser incluidas las formaciones Bocas y Jordán del Jurásico Temprano. Las formaciones Bocas y Jordán presentan tobas y material volcánico acido coetáneo con la sedimentación (Ward et al., 1973). La presencia de sedimentos lacustres en estas formaciones podría haber creado las condiciones para la generación de estructuras almohadilladas en los basaltos.

El volcanismo Jurásico es episódico en la Cordillera Oriental, sin embargo hacia el Valle del Magdalena se hace más importante y se reporta en las formaciones Norean (Clavijo, 1995) y La Mojana (Geyer, 1982) del Jurásico. El evento de volcanismo Triásico - Jurásico se extendió por la margen occidental de Sudamérica desde el Ecuador, Valle Superior del Magdalena (Mojica y Kammer, 1995), Sierra Nevada de Santa Marta (Tschanz, 1969) y Península de la Guajira (Radelli, 1960).

Los basaltos de la Formación Nogontova fueron formados sobre basamento continental constituido por rocas metamórficas del Macizo de Santander. Las coladas basálticas de esta unidad debieron fluir sobre sedimentos blandos y bajo el agua como lo indican las estructuras almohadilladas. La naturaleza geoquímica de los basaltos de la Formación Nogontova y su edad absoluta será clave para identificar el ambiente geodinámico de la unidad.

\section{AGRADECIMIENTOS}

Expresamos nuestros agradecimientos a la Universidad de Caldas, Departamento de Ciencias Geológicos por el apoyo en la práctica extramural de la actividad académica Geología de campo I y a los estudiantes del grupo 12, Daniel Quiroz, Lucney Mendoza y Sergio Gómez quienes contribuyeron con labores de campo y recolección de muestras. A los evaluadores Doctor Francisco José Pérez Torrado de la Universidad de Las Palmas de Gran Canaria y al revisor(a) anónimo(a) por sus valiosas sugerencias.

\section{REFERENCIAS}

Acosta, J., y Solano, O. 1999. Geología del Departamento de Boyacá. Escala: 1.250.000: INGEOMINAS. 
Ballard, R.D., Holcomb, R.T., and van Andel, T.H. 1979. The Galapagos Rift at $868 \mathrm{~W}$ : 3. Sheet flows, collapse pits, and lava lakes of the rift valley. Journal of Geophysical Research, 84: 5407-5422.

Campbell, C.J. 1962. A section through the Cordillera Oriental of Colombia between Bogotá and Villavicencio. Colombian Society of Petroleum, Geology and Geophysic, pp. 1-29.

Cediel, F. 1968. El Grupo Girón, una molasa mesozoica de la Cordillera Oriental. Boletín Geológico INGEOMINAS, 16 (1-3): 5-96.

Clavijo, J. 1995. Mapa Geológico de Colombia. Plancha 75-Aguachica, escala 1:100.000: INGEOMINAS.

Dickey, P.A. 1941. Pre-Cretaceous sediments in Cordillera Oriental of Colombia: American Association of Petroleum Geologist Bulletin, 25: 1789-1795.

Etayo-Serna, F., Barrero, D., Lozano, H.Q., Espinosa, A., González, H., Orrego, A., Ballesteros, I.T., Forero, H.O., Ramírez, C.Q., Zambrano-Ortiz, F., Duque-Caro, H., Vargas, R.H., Núñez, A., Álvarez, J.A., Ropain, U.C., Cardozo, E.P., Galvis, N., Sarmiento, L.R., Albers, J.P., Case, J.E., Singer, D.A., Bowen, R.W., Berger, B.R., Cox, D.P., and Hodges, C.A. 1986. Mapa de terrenos geológicos de Colombia. Publicaciones especiales INGEOMINAS, 1, 235p.

Geyer, O.F. 1982. Comparaciones estratigráficas y faciales en el Triásico Norandino. Geología Norandina, 5: 27-31.

Goldsmith, R., Marvin, R., and Mehnert, H. 1971. Radiometric ages in the Santander Massif, Eastern Cordillera, Colombian Andes. US Geological Survey Professional Paper, 750: 41-49.

Hedberg, H.D. 1931. Standard stratigraphic section of the Department of Santander, Colombia (inédito). Venezuela Gulf Oil Company.

Horton, B.K., Saylor, J.E., Nie, J., Mora, A., Parra, M., Reyes-Harker, A., and Stockli, D.F. 2010. Linking sedimentation in the northern Andes to basement configuration, Mesozoic extension, and Cenozoic shortening: evidence from detrital zircon $\mathrm{U}-\mathrm{Pb}$ ages, Eastern Cordillera, Colombia. Geological Society of America Bulletin, 122 (9-10): 1423-1442.

Jones, J. G. 1969. Pillow lavas as depth indicators. American Journal of Science, 267: 181-195.
Kammer, A., and Sanchez, J. 2006. Early Jurassic rift structures associated with the Soapaga and Boyaca faults of the Eastern Cordillera, Colombia: sedimentological inferences and regional implications. Journal of South American Earth Sciences, 21(4): 412-422.

Kennish, M.J., and Lutz, R.A. 1998. Morphology and distribution of lava flows on mid-ocean ridges: a review. Earth Science Reviews, 43: 63-90.

Langenheim, R.L. 1954. Preliminar report on the stratigraphy of the Girón Formation in Santander and Boyacá. Informe 1011. Instituto Geológico Nacional.

Le Maitre, R.W., Streckeisen, A., Zanettin, B., Le Bas, M.J., Bonin, B., and Bateman, P. 2002. Igneous rocks: a classification and glossary of terms: recommendations of the International Union of Geological Sciences Subcommission on the Systematics of Igneous Rocks, Cambridge, Cambridge University Press, 252p.

Maze, W.B. 1984. Jurassic La Quinta Formation in the Sierra de Perijá, northwestern Venezuela: Geology and tectonic environment of the red beds and volcanic rocks. In Bonini, W.E., Hargraves, R.B., and Shagam, R., (Eds.), Geological Society of America, Volume Memoir 162: Boulder, pp. 263-284.

Mojica, J., Y Franco, R. 1992. Estructura y evolución tectónica del Valle Medio y Superior del Magdalena: Geología Colombiana, 17: 41-64.

Mojica, J. C., Y Kammer, A. 1995. Eventos Jurásicos en Colombia: Geología Colombiana, 19: 165-172.

Moore, J.G. 1965. Petrology of deep-sea basalt near Hawaii: American Journal of Science, 263: 40-52.

Moore, J.G., and Schilling, J.G. 1973. Vesicles, water and sulphur in Reykjanes Ridge basalts. Contributions to Mineralogy and Petrology, 41: 105-118.

Morales, L.G., Podesta, D.J., Hatfield, W.C., Tanner, H., Jones, S.H., Barker, M.H.S., O’Donoghue, D.J., Mohler, E.P., Dubois, E.P., Jacobs, C., and Goss, C.R. 1958. General geology and oil occurrences of Middle Magdalena Valley, Colombia. In Weeks, L.G. (Ed.). Habitat of Oil, Symposium: Tulsa, American Association of Petroleum Geologists, pp. 641-695.

Moreno-Sánchez, M., Gómez-Cruz, A.d.J., and Castillo-González, H. 2005. La "Formación Floresta Metamorfoseada" (sensu Ward et al., 1973) no es la Formación Floresta sin metamorfosear. X Congreso Colombiano de Geología, Bogotá, Memorias CD, pp. 1-7. 
Moreno-Sánchez, M., Gómez-Cruz, A.d.J., and CastilloGonzález, H. 2008. Ocurrencia de fósiles Paleozoicos al este de la parte norte de la Cordillera Central y discusión sobre su significado geológico. Boletín de Ciencias de la Tierra, 22: 39-47.

Radelli, L. 1960. El basamento cristalino de la Península de La Guajira. Boletín Geológico del Servicio Geológico Nacional, 8 (1-3): 5-23.

Remy, W., Remy, R., Pfefferkorn, H.W., Volkheimer, W., and Rabe, E. 1975. Neueinstufung der Bocas-Folge (Bucaramanga, Kolumbien) in der unteren Jura anhand einer Phlebopteris-branneri- und Classopollis-Flora: Argumenta Paleobotanica, 4: 55-75.

Royero, G.J.M., y Vargas, H.R. 1999. Geología del Departamento de Santander. Escala, 1: 300.000. INGEOMINAS.

Tschanz, C.M. 1969. Mapa Geológico de reconocimiento de la Sierra Nevada de Santa Marta, Colombia. Escala 1:200.000. INGEOMINAS - USGS.

Vargas, R.H., Arias, A.T., Jaramillo, L.C., and Téllez, N. 1984. Geología de la plancha 136 Málaga. Escala 1:100.000: INGEOMINAS.

Ward, D., Goldsmith, R., Cruz, B., and Restrepo, H. 1973. Geología de los cuadrángulos H-12, Bucaramanga y H-13, Pamplona, Departamento de Santander: Boletín Geológico, 21(1-3): 1-132.

Trabajo recibido: junio 16 de 2015

Trabajo aceptado: octubre 23 de 2015

Manuscrito publicado en internet: noviembre 23 de 2015 